\title{
Curriculum development to improve student understanding of rolling motion
}

\author{
Sheh Lit Chang and Peter S. Shaffer \\ Department of Physics, University of Washington, Seattle, WA 98195-1560
}

\begin{abstract}
Research has led to the identification of some specific difficulties that students have in understanding the motion of an object that is rolling without slipping. At the University of Washington, we have been building on this work and are developing and testing a tutorial based on a relative motion approach to teaching rolling motion. Results from questions on this topic, administered before and after tutorial instruction, suggest that although this approach seems fruitful, additional research is needed to identify what is needed to help many students. The tutorial and results from several questions are presented to illustrate the instructional sequence and ongoing assessment.
\end{abstract}

\section{INTRODUCTION}

The introduction of rolling motion in introductory mechanics allows students to describe real-life, rigid-body motions. Until this point in a typical course, they have studied idealized systems involving either translation or rotation, but not both. The study of rolling motion has another benefit; it gives students an opportunity to apply concepts they have previously studied, such as relative motion, to more complicated situations. However, research indicates that students often have difficulty in applying Galilean relativity to rolling motion after standard instruction. Moreover, the difficulties can persist even after advanced study of mechanics [1].

A possible approach to improving student learning of rolling motion is to focus instruction explicitly on those concepts and reasoning skills that present difficulty to students. López suggested teaching rolling motion by introducing a definition of no-slip motion based on a relative motion approach [2]. For example, the instantaneous velocity of a point, $\mathrm{A}$, on a rotating rigid body in the reference frame of the Earth can be determined using the Galilean transformation of velocities: $\vec{v}_{\mathrm{A}}^{\mathrm{E}}=\vec{v}_{\mathrm{A}}^{\mathrm{CM}}+\vec{v}_{\mathrm{CM}}^{\mathrm{E}}$, where $\vec{v}_{\mathrm{A}}^{\mathrm{E}}$ is the velocity of point $\mathrm{A}$ in the Earth frame; $\vec{v}_{\mathrm{A}}^{\mathrm{CM}}$ is the velocity of point $\mathrm{A}$ in the center of mass frame; and $\vec{v}_{\mathrm{CM}}^{\mathrm{E}}$ is the velocity of the center of mass in the Earth frame. If the object is rolling without slipping, the center of mass velocity $\vec{v}_{\mathrm{CM}}^{\mathrm{E}}$ and the angular velocity $\omega$ are related through the no-slip condition $\left(v_{\mathrm{CM}}=R \omega\right)$.

To test the utility of using relative motion to teach rolling without slipping, we are designing a tutorial and examining its effect on student learning. The focus is not only on curriculum development, but also on extending the research base on student learning of rolling motion and related concepts. We are also trying to determine the extent to which student understanding of relative motion in general might be impacted.

\section{CONTEXT FOR RESEARCH}

This investigation is being carried out in the mechanics courses that are part of the calculus-based introductory sequence at the University of Washington (UW). Each week, these courses have three 50-minute lectures, a 50-minute small-group section using Tutorials in Introductory Physics [3], and a required 110-minute lab. In the tutorials, groups of between 3 to 5 students work together through carefully structured worksheets. Teaching Assistants (TAs) move from group to group using semi-Socratic methods to respond to student questions. A more detailed description of the tutorials, their implementation, and the preparation of TAs can be found in prior papers by our group [4].

The impact of the tutorials on student learning is assessed, in part, through comparisons of performance on 'pretest' and post-test questions. Pretests are administered online, usually after relevant lecture instruction, although they sometimes are given before any university instruction. Post-tests are given on course examinations after students have also worked through the tutorials and completed relevant tutorial homework. This investigation also includes results from a preliminary investigation in which the 'pretest' questions were given on a final exam prior to the implementation of the tutorial.

\section{PRELIMINARY INVESTIGATION}

The preliminary investigation, conducted before the tutorial was developed, included administering to students several questions from the Rotational and Rolling Motion Conceptual Survey (RRMCS) [1]. One of these is based on the motion of a point on the rim of a wheel that is rolling without slipping on a surface. (See Figure 1.) It was given on the final examination in a class at the UW after only standard instruction on rolling and slipping. It was used to examine student performance at the UW after lecture instruction and to compare these results with those from the original study at another university [1].

In the question, students are asked to find the instantaneous velocity of point 2 on the rim of the wheel relative to the reference frame of the road (Earth). The correct answer, option B, can be obtained using one of two approaches. Students could use the Galilean transformation of velocities by adding the velocity of the center-of-mass of the wheel in the road frame to the velocity of point 2 in the frame of the wheel $\left(e . g ., \vec{v}_{2}^{\mathrm{E}}=\vec{v}_{2}^{\mathrm{CM}}+\vec{v}_{\mathrm{CM}}^{\mathrm{E}}\right)$. Note that the no- 
Table 1: Student answers for the rolling task shown in Figure 1. The results include students from a prior study at another university; from the UW before and after only lecture instruction; and from after tutorial instruction.

\begin{tabular}{llcccc}
\hline Year/ \\
Quarter & Population and extent of instruction & \multirow{2}{*}{$N$} & \multicolumn{3}{c}{ Answers } \\
\cline { 5 - 6 } 2005 & Rimoldini and Singh (after lecture) [1] & 559 & $\mathbf{3 7 \%}$ & $48 \%$ & $15 \%$ \\
$2017 \mathrm{Au}$ & UW Final Exam (after lecture; without tutorial) & 648 & $\mathbf{3 5 \%}$ & $54 \%$ & $11 \%$ \\
$2018 \mathrm{Wi}$ & UW Pretest (before lecture; without tutorial) & 240 & $\mathbf{2 5 \%}$ & $65 \%$ & $10 \%$ \\
$2018 \mathrm{Sp}$ & UW Final Exam (after lecture and tutorial) & 157 & $\mathbf{7 3 \%}$ & $23 \%$ & $4 \%$ \\
\hline
\end{tabular}

slip condition means that $\vec{v}_{\mathrm{CM}}^{\mathrm{E}}$ and $\vec{v}_{2}^{\mathrm{CM}}$ have equal magnitude. An alternative approach is to imagine the (cycloidal) trajectory of point 2 in the frame of the Earth and to take the tangent to the curve at the instant shown.

A rigid wheel of radius $R$ rolls without slipping on a horizontal road. The linear velocity of the center of the wheel with respect to the road is $\vec{v}$ and the angular speed is $\omega$. What is the direction of the instantaneous velocity of point 2 with respect to the road? Explain.

A. $\searrow$ B. $\nearrow$ C. $\rightarrow$ D. $\uparrow$ E. Zero vector

Figure 1: Question asking students for the direction of the velocity of a point on the rim of a rigid body rolling without slipping. Students are asked to explain their answers in the pretest, but not required to explain in post-test. (Taken from the RRMCS [1].)

The results from Rimoldini and Singh's study and those from the UW (both after only standard instruction) are shown in the first two rows of Table 1, respectively. In each case, the proportion of students who answered correctly (option B) is about $35 \%$. The most common incorrect answer, given by about half of each population, was to say that $\vec{v}_{2}^{\mathrm{E}}$ is directly upward (choice D). This answer corresponds to the direction of the velocity of point 2 in the frame of the center-of-mass of the wheel. Student explanations suggested that some were reasoning explicitly based on the center-of-mass frame of the wheel, while others were answering based only on the velocity being tangent to the wheel at the instant shown.

The third row in Table 1 shows results from the same task given at the UW before any lecture instruction. (The timing of the tutorial in that quarter was such that the pretest came before lecture instruction.) The percentage of correct answers in this case was about $25 \%$. Since the variation we typically see on pretests from quarter to quarter and from class to class is typically about $\pm 5 \%$ [5], this result suggests that standard lecture instruction results in something like a $10 \%$ improvement on the task.

\section{OUTLINE OF TUTORIAL}

The tutorial was designed to help students understand rolling without slipping by guiding them through a relative motion approach. It starts in one dimension, then progresses to two dimensions. Physical situations that that research suggested could elicit student errors were used so that any tendency to reason incorrectly could be addressed explicitly.

The first section of the tutorial guides students through a review of the Galilean transformation of velocities for the case that all the motions are collinear. Students are shown a ground-based observer who is observing a box being pushed across a moving train car. They are asked to determine the three relevant displacements and to relate these vectors both diagrammatically and mathematically. They then relate the displacements with the corresponding velocities and express the results in terms of velocity.

In Section 2, students consider the motion of a hoop of radius $R$ that is moving to the right through the air while spinning clockwise with an angular velocity $\omega$. They first draw a vector to represent the velocity of the center of mass of the hoop in the ground frame. Next, they are asked to find the following velocities for the center-of-mass frame: (1) the center of mass of the hoop, (2) the lowest point on the rim and (3) the highest point on the rim. They apply the Galilean transformation of velocities in two dimensions to find the range of possible magnitudes and directions of the instantaneous velocities of all three points in the ground frame.

Section 2 concludes by leading students to recognize that without additional information (i.e., the relationship between the velocity of the center-of-mass in the ground frame and the angular velocity, $(\omega)$, it is not possible to determine whether in the ground frame the lowest point on the hoop has a velocity to the right, to the left, or zero.

The final section of the tutorial starts with a description of how rolling with slipping compares to rolling without slipping for a cylinder on a table. Students are explicitly told how these compare in terms of the relative motion of the points on the cylinder and table that are instantaneously in contact. 
Based on the descriptions, students are then asked to apply the idea of no-slip motion to determine the condition for pure rolling motion (i.e., $\left.v_{\mathrm{CM}}=R \omega\right)$. They then predict how the motion of a cylinder changes with time for three different initial conditions: $v_{\mathrm{CM}}=R \omega, v_{\mathrm{CM}}>R \omega$, and $v_{\mathrm{CM}}<R \omega$.

At the end of the tutorial, the TAs perform a demonstration so that students can check their predictions. Students record their observations, draw free-body diagrams for the cylinders, and use the diagrams to account for the observed changes in motion.

In the tutorial homework, students apply what they have learned to determine the magnitudes and directions of the velocities of all three points 1, 2, and 3 on the wheel shown in Figure 1. On another question, students are shown a board on top of three cylinders and told that a force is pushing the board such that there is no slipping between the board and cylinders or between the board and the ground. They are then asked to relate the velocities and accelerations of the board and the center-of-mass of the cylinder.

\section{ASSESSING THE IMPACT OF THE TUTORIAL}

Various tasks are being used to assess the effect of the tutorial on student understanding of rolling without slipping. Two such tasks are discussed in this section: (1) the rolling motion task from the RRMCS shown in Figure 1 and (2) an extension of that task that asks students to rank the velocities of three points on the wheel. (The second task was also part of the RRMCS.)

\section{Task 1: Rolling motion task in RRMCS}

Variations of Task 1 (Fig. 1) have been used as part of the assessment of the tutorial. In Spring 2018 it was on the final exam after students had completed the tutorial and had gotten back their graded homework. The results are shown in the last row of Table 1.

About $70 \%$ of the students answered correctly, which corresponds to a gain of around $40 \%$ compared to the pretest. The most common error was for students to state that the velocity is tangent to the wheel at the instant shown, which corresponds to the direction of the velocity in the center of mass frame. However, the on the post-test, the prevalence of this error was reduced to about $25 \%$ of the class compared to about $50 \%$ after lecture instruction.

\section{Task 2: Ranking task from RRMCS}

The second question used to assess the tutorial is also based on a question from the RRMCS. As for Task 2, the question is based on the situation in Figure 1. Student are asked to rank the speeds in the frame of the road of the three points 1, 2, and 3 on the rim of the wheel. Points 1 and 3 are at the bottom and top of the wheel, respectively, at the instant shown. Point 2 is the same point as in Task 1.

To answer correctly, students need to recognize that since the wheel is rolling without slipping, point 1 (at the bottom of the hoop) has zero velocity relative to the road. Point 3 has twice the velocity of the center-of-mass of the wheel $\left(v_{\mathrm{CM}}\right)$ and point 2 has a speed of $2 \sqrt{v_{\mathrm{CM}}}$. Thus, the correct ranking is $v_{3}>v_{2}>v_{1}$.

Variants of this question were administered to students in three classes at three different points during instruction: prior to any instruction on rolling, after lecture instruction only, and after lecture plus tutorial instruction. The percentages of students answering correctly were about $15 \%, 45 \%$, and $75 \%$ respectively. (The results from after only lecture instruction are similar to those documented in Ref. 1.)

The most common incorrect answer given by students in all the courses was that all three velocities are equal. Since the wheel is clearly rolling, this suggests that the rolling without slipping condition is very hard for students, especially before instruction on this topic.

\section{ADDITIONAL INVESTIGATION}

On task 1 (Fig. 1), many of the students who chose the upward direction of velocity for point $B$ in the ground (CM) frame explained that the velocity should be tangent to the circle or stated that this direction corresponds to the direction of the tangential velocity. These explanations suggested that the students might be basing their answer solely on the shape of the wheel and not thinking about the trajectory of that point on the wheel in the ground frame. We were interested in how students would answer if they were shown the trajectory.

Two different pretests were developed and administered in during Spring 2018. (See Figure 2.) Task 2A was given in two lecture sections; task $2 \mathrm{~B}$ was given in a third section. (Results from other pretests given to all three sections were similar and suggest that the populations are essentially equivalent.)

Both questions are based on the same trajectory. In task 2A, students were shown only the trajectory, without reference to how it was produced. In Task $2 \mathrm{~B}$, students were told the trajectory is that produced by point on the rim of a rolling cylinder. They were shown the cylinder at an instant and the trajectory over a period of time. In both cases, students were asked to pick the vector that represents the instantaneous velocity at point $\mathrm{M}$. The correct answer in both cases is option B.

Only about $60 \%$ of the students chose the correct answer on Task 2B, while $90 \%$ did so on Task $2 \mathrm{~A}$. Most who answered correctly gave a correct explanation, for example that the vector is tangential to the curve.

On task $2 \mathrm{~B}$, about $30 \%$ of the students chose the incorrect option D (directly upward). Most of them explained their choice by saying that the arrow is tangent to the cylinder or simply that this is the direction of the velocity for circular 
motion. The results from tasks $2 \mathrm{~A}$ and $2 \mathrm{~B}$ taken together indicate that although most students can use the tangent to the trajectory to find the direction of the instantaneous velocity, for many, the 'source' of the motion (the cylinder) seems to serve as a distractor.

Task 2A: A particle travels from $\mathrm{P}$ to $\mathrm{Q}$ along a curved trajectory shown below. When the particle is at point $\mathrm{M}$, which of the following options best represents the direction of its instantaneous velocity?

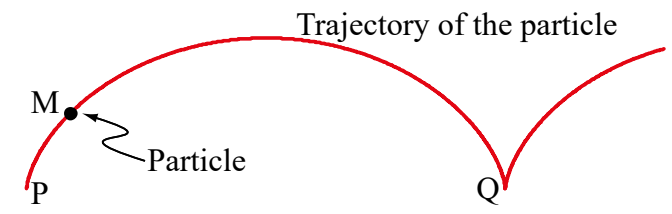
A.
B.

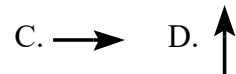
E. Zero vector

Task 2B: A cylinder rolls without slipping on a surface as shown below. The trajectory of a point on the rim of the cylinder (marked by the black dot) is traced out in dashed line. When the dot is at point $\mathrm{M}$, which of the following options best represents the direction of its instantaneous velocity?

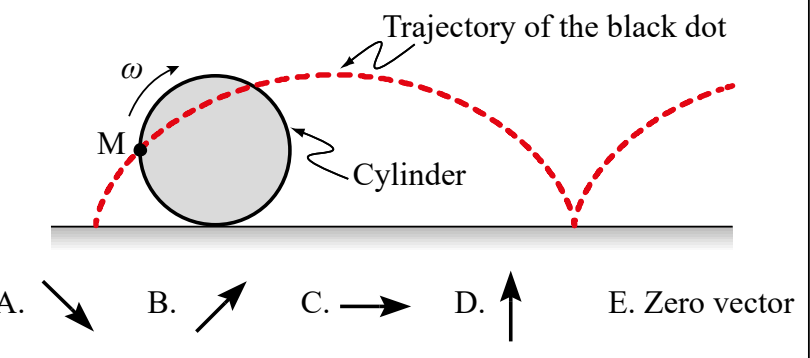

Figure 2: Questions used to determine the effect of showing students the trajectory of a point on the wheel.

A comparison of the results from task 1 when administered as a pretest $(25 \%$ correct) and task $2 \mathrm{~A}(90 \%$ correct) suggests that the presence of the trajectory strongly affects student ability to identify the correct direction for the velocity.

\section{DISCUSSION AND FUTURE WORK}

The results from the research presented in this paper are consistent with those from prior research on rolling motion. Most students enter introductory physics courses with incorrect ideas about the rotational motion of rigid bodies and these are not addressed by traditional instruction. Many students fail to construct a functional understanding of the constraint $v_{\mathrm{CM}}=\omega R$ for an object that is rolling without slipping and do not recognize that the point of contact for the object has zero velocity relative to the constraining surface. When asked to find the velocity of a point on a rolling object, students often seem to be inconsistent in their choice of reference frame. In other cases they seem to use the shape of the object at a given instant to find the velocity and do not consider the motion over an extended interval of time.

The tutorial that we are developing to help students reason about rolling motion uses an approach based on reference frames and relative motion. It was constructed to address many of the difficulties that had been identified. Although it seems to be somewhat effective, we believe that more can be done. After instruction, about one quarter of the students still answer incorrectly on relatively basic questions like the one shown in Figure 1. We also have evidence from other questions (not discussed) that the tutorial does not seem to impact student performance on some standard relative motion questions that often appear in textbooks (e.g., the motion of a boat in a river).

We are in the process of conducting additional research that includes examining student understanding of instantaneous velocity. The next phase of curriculum development is targeted at helping students make connections between the trajectory of an object and relative motion concepts.

\section{ACKNOWLEDGEMENTS}

We would like to thank UW faculty members Suzanne Brahmia, Jason Detwiler, Kai-Mei Fu, R. Daryl Pedigo, Gray Rybka, Nikolai Tolich, and Kazumi Tolich for welcoming this research in their courses. This work has been supported in part by the National Science Foundation under Grant No. DUE 1022449.

[1] Rimoldini, L. G. and Singh, C., Phys. Rev. St Phys. Educ. Res. 1, 010102, (2005).

[2] López, M., Eur. J. Phys. 24, 553-562, (2003)

[3] L. C. McDermott et al, Tutorials in Introductory Physics, Preliminary 2nd edition (2011).

[4] L. C. McDermott, Am. J. Phys., 73, 921 (2005).

[5] Heron, P. R. L., Phys. Rev. St Phys. Educ. Res. 11, 010102, (2015). 